\title{
Range and Velocity Ambiguity Mitigation Techniques for the WSR-88D Weather Radar
}

\author{
Sebastián M. Torres \\ Cooperative Inst. for Mesoscale Meteorological Studies \\ The University of Oklahoma \\ Norman, OK \\ sebastian.torres@noaa.gov
}

\author{
Dusan S. Zrnić \\ National Severe Storms Laboratory \\ NOAA \\ Norman, OK \\ dusan.zrnic@noaa.gov
}

\begin{abstract}
Several mechanisms are currently provided to alleviate effects of range overlaid echoes and velocity aliasing in the Weather Surveillance Radar-1988 Doppler (WSR-88D). However, due to limitations in these techniques, observation of severe weather phenomena is significantly impaired. This paper presents results from a multi-year study at the National Severe Storms Laboratory dealing with methods to mitigate the effects of range and velocity ambiguities in the WSR-88D.
\end{abstract}

Keywords-Doppler weather radar; range and velocity ambiguity mitigation; systematic phase coding; staggered pulse repetition time

\section{INTRODUCTION}

It is well known that for Doppler radars transmitting uniformly spaced pulses there is a coupling between the maximum unambiguous range $\left(r_{a}\right)$ and the maximum unambiguous velocity $\left(v_{a}\right) ; r_{a}$ or $v_{a}$ can only be increased at the expense of a proportional decrease in the other [1]. This is a fundamental limitation for the observation of severe weather. Various schemes have been proposed to mitigate the effects of ambiguities, but few have been tested and even fewer are available on operational Doppler weather radars. Examples of these are random phase coding and dual pulse repetition frequency (PRF), which have been tested and are commercially available $[2,3]$.

Two mechanisms are currently provided to alleviate effects of range overlaid echoes and velocity aliasing in the Weather Surveillance Radar-1988 Doppler (WSR-88D). At the lowest elevations, the WSR-88D performs two scans at each elevation angle. Each of these pairs of scans is usually referred to as a "split cut". The first scan uses a long pulse repetition time (PRT) and produces power (reflectivity) estimates up to 460 $\mathrm{km}$. Doppler velocity estimates from this scan are almost useless due to their low $v_{a}$ (about $9 \mathrm{~m} \mathrm{~s}^{-1}$ ). The second scan at the same elevation angle uses a short PRT with $r_{a}=148 \mathrm{~km}$ and produces (possibly range folded) $v_{a}$ in the range $\pm 28 \mathrm{~m} \mathrm{~s}^{-1}$. Signal processing algorithms in the radar data acquisition (RDA) subsystem of the WSR-88D use the long-PRT power data to position velocity estimates from the short-PRT scan to the range location of the strongest trip echo. At intermediate elevation angles, where clutter rejection requirements are less stringent, the WSR-88D reduces the time by running just one scan in the "batch mode" whereby long-PRT and short-PRT

The Radar Operations Center of the National Weather Service provided partial support for this work through a Memorandum of Understanding. Funding for this research was provided under NOAA-OU Cooperative Agreement \#NA17RJ1227. blocks of pulses are interlaced. Analogously to the split cut processing, powers are obtained from pulses at the long PRT $\left(r_{a}>230 \mathrm{~km}\right)$ and velocities from the short-PRT batch $\left(v_{a}=28\right.$ $\mathrm{m} \mathrm{s}^{-1}$ ) using the range unfolding algorithm mentioned above. However, this algorithm fails in regions where the overlaid trip powers in the short-PRT scan are within $5 \mathrm{~dB}$ of each other. Therefore, the WSR-88D cannot recover velocities from the weaker trips or resolve strong overlays. Doppler velocity displays characterize these failures by encoding locations of such overlaid powers with a purple color, normally referred to as the "purple haze".

Over the last decade, two techniques have emerged as viable candidates to address the mitigation of range and velocity ambiguities in the WSR-88D thus reducing the amount of purple haze obscuration currently encountered during the observation of severe phenomena [4]. These are: systematic phase coding and staggered PRT. The two techniques are complementary since they offer advantages at specific elevation angles; hence, they can be simultaneously incorporated into the same volume coverage pattern (VCP). This paper describes the proposed scanning strategy, and shows preliminary results on weather data collected with the KOUN research radar in Norman, Oklahoma. Limitations and tradeoffs of each technique are discussed, and examples comparing their performance are presented. Results from the analyzed events demonstrate the ability of systematic phase coding and staggered PRT to effectively mitigate range and velocity ambiguities in future enhancements of the United States network of weather surveillance radars.

\section{RANGE AND VELOCITY AMBIGUity MitigAtion}

Unlike point scatterers (e.g., airplanes), meteorological scatterers are distributed continuously and produce semicoherent signals with very large dynamic range (about $100 \mathrm{~dB}$ ). Consequently, weather radars are overwhelmed with velocity ambiguities and overlaid echoes (i.e., echoes that return after a subsequent pulse is transmitted). Unfortunately, complete resolution of range and velocity ambiguities is not possible; the two techniques described herein aim, at best, for a reduction of their effects.

\section{A. SZ Phase Coding}

Sachidananda and Zrnić [5] proposed the SZ phase code as 
a better alternative to random codes. SZ phase coding is similar to random phase coding except that the transmitted pulses are phase-modulated with a systematic code consisting of $M$ phases that repeat periodically. From the family of $\mathrm{SZ}(n / M)$ codes, the SZ(8/64) code gives the best performance in terms of recovery of overlaid trips [6]. This code exhibits properties that make it attractive for the separation (in the spectral domain) of overlaid signals. If the received signal is cohered for a given trip, the spectra of all out-of-trip echoes consist of evenly spaced replicas of their corresponding coherent spectra. Hence, out-of-trip echoes do not bias the mean Doppler velocity estimate of the coherent signal. Once the velocity is recovered for the strong-trip, the coherent signal is notched out such that the two least contaminated replicas of the out-of-trip (i.e., the weak trip) echo remain. These two replicas are enough to reconstruct (cohere) the weak-trip echo and recover its mean Doppler velocity. Recovery of strong and weak trip signals can proceed in a stand-alone manner or with the aid of an extra scan (at the same elevation angle) using a long PRT. Although the latter requires an additional scan similarly to the split cuts described above, long PRT data provides non-overlaid power information that is essential in the determination of overlaid trips and their relative strengths. Having the long-PRT information available greatly simplifies the SZ decoding algorithm and leads to better overlay recovery.

\section{B. Staggered PRT}

The staggered PRT technique was first proposed in the context of weather surveillance radars by Sirmans et al. [7]. With this technique, transmitter pulses are spaced at alternating PRTs, $T_{1}$ and $T_{2}$, and pulse-pair autocorrelation estimates are made independently for each PRT. These estimates are suitably combined so that the effective maximum unambiguous velocity can be extended to $v_{a}=m \lambda / 4 T_{1}=n \lambda / 4 T_{2}$ [8], where the stagger PRT ratio is given by $T_{1} / T_{2}=m / n$ ( $m$ and $n$ are integers) and $\lambda$ is the transmitter wavelength. In addition, $r_{a}=c T_{1} / 2$, corresponding to the shorter PRT ( $c$ is the speed of light). At the core of this technique is the velocity dealiasing algorithm; to determine the Nyquist interval of the true velocity, it uses the fact that Doppler velocities obtained from the short and long PRTs alias in different ways.

\section{RESULTS ON WEATHER DATA}

SZ phase coding and staggered PRT techniques have been applied to several events of time series data collected by the KOUN radar in Norman, OK. The case in Fig. 1 was obtained on April 06, 2003 at approximately 04:30 GMT. KOUN ran a scan at $1.5 \mathrm{deg}$ using a long uniform PRT followed by three scans at the same elevation angle. The first of these scans (top panel) used the standard legacy processing with a uniform PRT $T=986.7 \mu$ s yielding $v_{a}=28.08 \mathrm{~m} \mathrm{~s}^{-1}$ and $r_{\mathrm{a}}=148 \mathrm{~km}$. The second scan (middle panel) employs SZ phase coding with the same uniform PRT, and the third scan (bottom panel) uses the staggered PRT mode where $T_{1}=3.107 \mathrm{~ms}\left(r_{\mathrm{a} 1}=466 \mathrm{~km}\right)$ and $T_{2}=2.24 \mathrm{~ms}\left(r_{a 2}=336 \mathrm{~km}\right)$. Note that $T_{1} / T_{2}=0.72$ and the resulting composite maximum unambiguous velocity is $v_{a}=32 \mathrm{~m} \mathrm{~s}^{-1}$.

As expected, the legacy-type Doppler velocity display is significantly obscured by the "purple haze" which indicates the presence of unresolvable overlaid echoes. While velocity estimates agree fairly well in places where no overlay occurs, estimates obtained with the staggered PRT algorithm exhibit less speckling than with phase coding but can be erratic in places of low SNR. In addition, the performance of the simple ground clutter filter implemented in this version of the staggered PRT algorithm is inferior compared to the recursive ground clutter filter used in the WSR-88D with uniform PRT
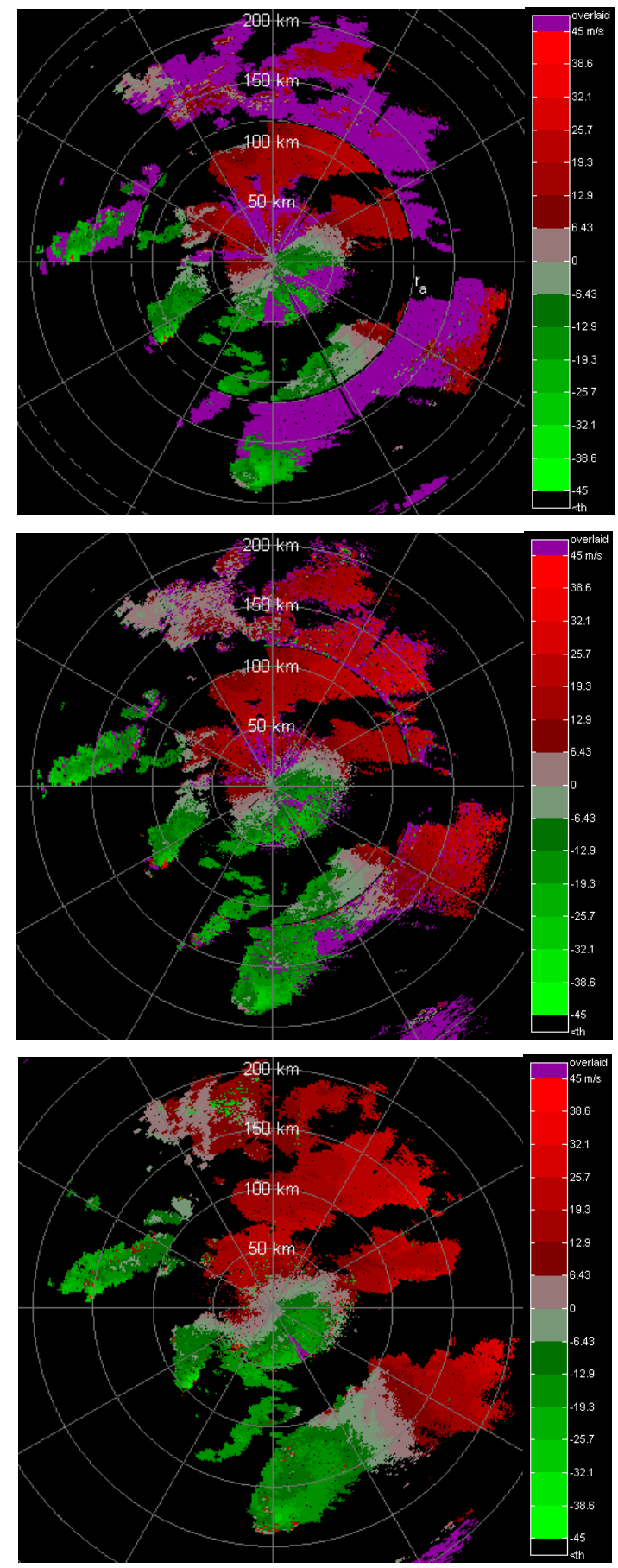

Figure 1. Doppler velocity displays of severe storms in central Oklahoma obtained using the legacy WSR-88D algorithms (top panel), SZ phase coding (middle panel), and staggered PRT (bottom panel). Purple denotes an unrecoverable velocity due to overlaid echoes. 
sequences. Evidence of this is the velocity bias towards zero observed on range bins close to the radar when comparing the corresponding velocity fields.

Compared to the current processing in the WSR-88D, both phase coding and staggered PRT produce a significantly smaller amount of obscuration due to overlaid echoes. A quantitative comparison of obscuration and additional examples on real data will be shown during the presentation at the conference.

\section{COMPARISON OF TECHNIQUES}

SZ phase coding allows the use of a short PRT to achieve maximum unambiguous velocities that are large enough to avoid velocity aliasing problems. Nevertheless, the use of a short PRT leads to overlaid echoes. These are eventually separated in the spectral domain. Conversely, the staggered PRT technique uses longer PRTs to accomplish separation of overlaid echoes in the time domain. In this case, the maximum unambiguous Doppler velocity is extended by the velocity dealiasing algorithm as mentioned above.

SZ phase coding is conducive to efficient ground clutter filters and spectral processing, since it employs a uniform PRT. This is not the case for the staggered PRT technique, where the design of efficient ground clutter filters has been a challenge [9] and is still a topic under research. If it were not for ground clutter filtering, the staggered PRT would have an advantage over phase coding because it can completely eliminate range overlay. However, theory suggests that ground clutter filtering of staggered pulses would have $10 \mathrm{~dB}$ less rejection than similar filtering on uniform pulses $[10,11]$. This remains to be tested on real data. Another advantage of uniform pulse sequences is their suitability for spectral analysis, which in turn can improve data quality. Except at the highest elevations, overlaid echoes are unavoidable; thus, one can argue that while spectral analysis (on uniformly spaced sequences) could improve the quality of some data, there would still be areas with overlaid echoes. This is in contrast to staggered PRT, which is inferior for spectral analysis but can provide data completely free of overlaid echoes. With staggered PRTs, the unambiguous range for reflectivity fields is larger than for velocity fields. Therefore, it is possible to satisfy the WSR-88D requirements on the range for velocity measurements (currently $230 \mathrm{~km}$ ) and have no overlaid echoes in the reflectivity fields!

\section{Proposed Volume Coverage Pattern}

Volume coverage patterns currently used in the WSR-88D for the observation of severe weather employ split cuts at the lower elevation angles, the batch mode at intermediate elevations, and short uniform PRT at higher elevation angles where overlaid echoes are less likely. For future enhancements of the network, we propose similar VCPs whereby the second half of split cuts are replaced by SZ phase coding scans, and the staggered PRT technique is used both at intermediate and high elevation angles where requirements for ground clutter removal are less stringent. These changes are made such that that current update times and number of scans in a VCP are preserved [12].

\section{CONCLUSIONS}

Analysis on real data and simulations indicate that phase coding cannot eliminate the long PRT scan for reflectivity estimation if the estimates must be unambiguous to $460 \mathrm{~km}$. Phase coding is effective on uniform PRT sequences which are conducive to spectral analysis and good ground clutter filtering. Increase in clear range is at least twice the unambiguous range of the inherent uniform short PRT, but not all overlaid echoes can be separated. Further, multiple overlaid signals might also cause total loss of information. Staggered PRT can provide clear range and relatively large unambiguous velocity, but for large unambiguous range, the errors in spectral moments might be prohibitive. The main disadvantage of staggered PRT is that ground clutter filtering is less effective or more complex and that spectral analysis, although possible, is severely impaired by the non-uniform spacing of samples. The two methods are complementary and a volume coverage pattern has been suggested to take advantage of the benefits offered by each method; phase coding at the lowest elevations and staggered PRT at higher elevations. Qualitative examination of these data confirms that the VCP suggested by Sachidananda et al. [11, 12] would be free of range overlaid echoes at higher elevations (using the staggered PRT technique), and would provide good clutter filtering and decrease areas of unrecoverable signals at low elevation (using the SZ phase coding technique).

\section{REFERENCES}

[1] Doviak, R. J. and D. S. Zrnić, Doppler radar and weather observations. New York: Academic Press, 1993.

[2] Joe, P., and P. T. May, "Correction of Dual PRF Velocity Errors for Operational Doppler Weather Radars," J. Atmos. Oceanic Technol., vol. 20, pp. 429-442, 2003.

[3] Joe, P., D. Hudak, J. Scott, R. Passarelli, and A. Siggia, "Operational evaluation of range ambiguity resolution by phase diversity," 28th Conf. on Radar Meteorology, Austin, TX, pp. 250-251, 1997.

[4] Zrnić, D. and R. Cook, "Evaluation of techniques to mitigate range and velocity ambiguities on the WSR-88D," $18^{\text {th }}$ International Conf. on IIPS, Orlando, FL, paper 5.13, 2002.

[5] Sachidananda, M. and D. S. Zrnić, "Systematic phase codes for resolving range overlaid signals in a Doppler weather radar," J. Atmos. Oceanic Technol., vol. 16, pp. 1351-1363, 1999.

[6] Sachidananda, M., D. S. Zrnić, R. J. Doviak, and S. M. Torres, Signal design and processing techniques for WSR-88D ambiguity resolution, Part 2. NOAA/NSSL Report, 1998.

[7] Sirmans, D., D. Zrnić, and B. Bumgarner, "Extension of maximum unambiguous Doppler velocity by use of two sampling rates," 17th Conf. on Radar Meteorology, Seattle, WA, pp. 23-28, 1976.

[8] Torres, S. M., Y. F. Dubel, and D. S. Zrnic, "Design, implementation, and demonstration of a staggered PRT algorithm for the WSR-88D," J. Atmos. Oceanic Technol., in press.

[9] Sachidananda, M. and D. S. Zrnić, "An improved clutter filtering and spectral moment estimation algorithm for staggered PRT sequences," J. Atmos. Oceanic Technol., vol. 19, pp. 2009-2019, 2002.

[10] Sachidananda, M., D. S. Zrnić, and R. J. Doviak, Signal design and processing techniques for WSR-88D ambiguity resolution, Part 3. NOAA/NSSL Report, 1999.

[11] Sachidananda, M., D. S. Zrnić, and R. J. Doviak, Signal design and processing techniques for WSR-88D ambiguity resolution, Part 4. NOAA/NSSL Report, 2000.

[12] Sachidananda, M., D. S. Zrnić, and R. J. Doviak, Signal design and processing techniques for WSR-88D ambiguity resolution, Part 5. NOAA/NSSL Report, 2001. 